\title{
Delivery outcomes of patients with pre-eclampsia at 27 - 34 weeks' gestation at Tygerberg Hospital, Cape Town, South Africa: A retrospective observational study
}

\author{
S E Olivier, ${ }^{1}$ MB ChB; L de Waard, ${ }^{1}$ MB ChB, Dip Obst (SA), FCOG (SA), MMed (O\&G); C J B Muller, ${ }^{2}$ MComm, MSc, PhD; \\ G S Gebhardt, ${ }^{1}$ MB ChB, Dip Obst (SA), FCOG (O\&G), MMed (O\&G), MSc (Med Sci), PhD, MSc (Clin Epi) \\ ${ }^{1}$ Department of Obstetrics and Gynaecology, Tygerberg Hospital and Faculty of Medicine and Health Sciences, Stellenbosch University, \\ Cape Town, South Africa \\ ${ }^{2}$ Department of Statistics and Actuarial Science, Faculty of Economic and Management Sciences, Stellenbosch University, Stellenbosch, South Africa
}

Corresponding author: S E Olivier (elzanne.se@gmail.com)

\begin{abstract}
Background. Obstetricians are cognisant of the serious nature of hypertensive disorders in pregnancy. Despite a $17 \%$ overall reduction in maternal deaths in South Africa between 2011 and 2016, there was a 14\% increase in deaths due to hypertension. Delivery is the only known cure for pre-eclampsia, but the question regarding the safest route of delivery remains difficult to answer.

Objectives. To determine the success rate of induction of labour ( $\mathrm{IoL}$ ) in patients with early-onset pre-eclampsia with severe features (EOPES) before 34 weeks' gestation. Furthermore, the data from the induction group were compared with those of the caesarean delivery (CD) groups where patients were not eligible for IoL. Additional objectives were to identify variables that could influence the success rate, to determine whether any delivery method was associated with increased morbidity, to assess the short-term maternal and neonatal outcomes, and to make recommendations for future decision-making regarding delivery for women with EOPES.

Methods. In this single-institution retrospective observational study, all cases in which a decision for delivery was made before 34 weeks 0 days of gestation (or the infant's birthweight was $\leq 2000 \mathrm{~g}$ with uncertain gestation) at Tygerberg Hospital, Cape Town, between 1 January and 30 June 2017 were identified from the electronic birth register. The cohort fitting the inclusion criteria was subdivided into IoL and CD groups. Results. From a total of 3938 deliveries, 168 patients met the inclusion criteria. IoL was indicated in 55 cases, resulting in 20 vaginal deliveries (VDs) (36\%) and $35 \mathrm{CDs}$ (64\%). The remaining 113 patients were not candidates for IoL; of these, 89 required emergency CDs and 24 had semi-elective CDs. In the IoL group with abnormal umbilical artery Dopplers (UADs) there was $1 \mathrm{VD}$, and 4 CDs were performed for fetal compromise. Of cases with an estimated fetal weight (EFW) $\leq 3$ rd centile, emergency CD was required in $24(65 \%)$, and 8 (22\%) were considered for IoL, in 6 of which CD was required.

Conclusions. Of the EOPES population, $36 \%$ had successful IoL that culminated in VD. VD was more likely to occur with fetal growth appropriate for gestational age. The likelihood of CD increased if the UAD was abnormal, if the EFW was $\leq 3$ rd centile or if eclampsia was present. The decision to induce should be considered carefully in these circumstances.
\end{abstract}

S Afr Med J 2021;111(5):437-443. https://doi.org/10.7196/SAMJ.2021.v111i5.15201

Obstetricians are cognisant of the serious nature of hypertensive disorders in pregnancy, which remains a major stumbling block in the battle to save mothers. Despite a $17 \%$ overall reduction in maternal deaths in South Africa (SA) between 2011 and 2016, there was a $14 \%$ increase in deaths due to hypertension. ${ }^{[1]}$ Pre-eclampsia results in $0.4 \%$ of primigravidas giving birth before 34 weeks' gestation, and $8-10 \%$ of all preterm births result from hypertensive disorders. ${ }^{[2]}$ Delivery is the only known cure for pre-eclampsia, ${ }^{[3]}$ but the question regarding the safest route of delivery remains difficult to answer.

Along with the decision to induce labour, the chances of success should be considered. Factors such as an unfavourable cervical Bishop score and nulliparity were shown to decrease the chances of success. ${ }^{[3]}$ Preterm gestation and advancing maternal age also increase the risk of caesarean delivery (CD). Failure to progress and non-reassuring fetal heart rate (FHR) patterns are the most common indications for $\mathrm{CD} .{ }^{[4]}$ The Consortium on Safe Labor study ${ }^{[5]}$ observed that vaginal delivery (VD) occurred in $57-80 \%$ of women who underwent preterm induction of labour (IoL), and the success rate increased with an increase in gestational age. Parity and a favourable cervical score are the strongest predictors of successful VD. ${ }^{[5]}$
On the other side of the coin are the risks associated with $\mathrm{CD}$. In $\mathrm{SA}$, the case fatality rate due to bleeding during $\mathrm{CD}$ increased from 20.9/100 000 live births in $2002-2004$ to 33.6/100 000 in $2011-2013 .{ }^{[6]}$ The maternal mortality ratio (MMR, deaths per 100000 live births) was 165 for CD v. 53 for VD. At primary care level, the MMR was 110 for CD compared with 25 for VD. ${ }^{[1]}$ The total number of CDs in public health facilities in SA (according to the District Health Information System (DHIS)) increased from 742751 in 2002 to 1011305 in 2019. The CD rate increased from $16.1 \%$ to 23.2\% between 2002 and 2013 and to $27 \%$ in 2019 (DHIS data). ${ }^{[6]}$

\section{Objectives}

To determine the success rate of IoL in patients with early-onset preeclampsia with severe features (EOPES) before 34 weeks' gestation. Furthermore, the data from the induction group were compared with those of the CD groups where patients were not eligible for IoL. Additional objectives were to identify variables that could influence the success rate of IoL, to determine whether any delivery method was associated with increased morbidity, to assess the short-term maternal and neonatal outcomes, and to make recommendations for future decision-making regarding delivery for women with EOPES. 


\section{Methods}

In this single-institution retrospective observational study, all cases in which a decision for delivery was made before 34 weeks 0 days of gestation (or the infant's birthweight was $\leq 2000 \mathrm{~g}$ with an uncertain gestational age) at Tygerberg Hospital, Cape Town, SA, between 1 January and 30 June 2017 were identified from the labour ward electronic birth register. Ethics approval was obtained from the Health Research Ethics Committee of Stellenbosch University (ref. no. S18/09/186), and permission from Tygerberg Hospital was also obtained.

The inclusion criteria were gravid women with a singleton pregnancy admitted with confirmed or presumed pre-eclampsia or eclampsia, as defined by the International Society for the Study of Hypertension in Pregnancy, ${ }^{[7]}$ who required delivery at 27 weeks 0 days - 34 weeks 0 days of certain gestation or delivered an infant with a birthweight of $800-2000 \mathrm{~g}$ if gestation was uncertain. ${ }^{[8]}$ Cases with a gestational age $<27$ weeks 0 days or $>34$ weeks 0 days, with an estimated fetal weight (EFW) $<800 \mathrm{~g}$ or $>2000 \mathrm{~g}$ when gestation was uncertain, multiple pregnancies, confirmed spontaneous rupture of membranes before induction, and confirmed labour on arrival were excluded. The data were analysed and described using 95\% confidence intervals, and significance was defined as a $p$-value $<0.05$.

The cohort was subdivided into four groups: IoL-CD (IoL ending with CD), IoLVD (IoL ending with VD), emergency CD (CD for urgent maternal or fetal indications) and semi-elective CD (CD indicated due to an abnormal lie, previous uterine surgery or physician concern with regard to fetal condition). The following variables with the potential to influence the success of IoL were assessed: age, parity, body mass index (BMI), HIV status, previous $\mathrm{CD}$, cervical favourability, transfer and arrival blood pressure, EFW and umbilical artery Dopplers (UADs). A stillbirth or early neonatal death and the 1-minute and 5-minute Apgar scores were used to evaluate the short-term neonatal outcomes. Maternal complications associated with the delivery method during hospital stay were recorded.

\section{Results \\ Study population}

From a group of 3938 deliveries within the weight and gestational age cut-off, 168 patients who met the inclusion criteria were identified. IoL was indicated in 55 cases, resulting in $20 \mathrm{VDs}$ (36\%) and 35 CDs (64\%). The remaining 113 patients (67\% of the total group) were not candidates for induction; of these, 89 required emergency CD and 24 had scheduled emergency (semi-elective) CD.

\section{Demographic data}

The demographic characteristics of the study population (Table 1) reflect a homogeneous group with no statistically significant differences between the delivery groups in terms of maternal age, BMI, HIV status or parity.

\section{Population characteristics Blood pressure}

Transfer and arrival blood pressures are shown in Fig. 1. Compared with the other delivery groups, patients in the IoL-VD group had lower median systolic blood pressures on transfer from the referral institution and on arrival at Tygerberg Hospital, and they also had lower arrival diastolic blood pressures. Despite this observation, the intergroup differences were statistically insignificant (systolic blood pressure transfer $p=0.383$, systolic blood pressure arrival $p=0.8122$, diastolic blood pressure transfer $p=0.2452$, diastolic blood pressure arrival $p=0.4037$ ).

\section{Gestational age}

Median gestational age was 30 weeks 2 days at the time that delivery was indicated in the emergency CD group, 32 weeks 5 days for the IoL-VD group, 32 weeks 1 day for the IoLCD group, and 31 weeks 6 days for the semielective $\mathrm{CD}$ group. The median gestational age difference between the group who required emergency $\mathrm{CD}$ and the subgroup who underwent IoL with successful VD was 2 weeks 3 days $(p<0.001)$ (Fig. 2).

\section{Delivery indication}

Fetal distress was the most common feature indicating delivery, succeeded by the 34 weeks 0 days' gestation cut-off (which is the institution's endpoint for expectant

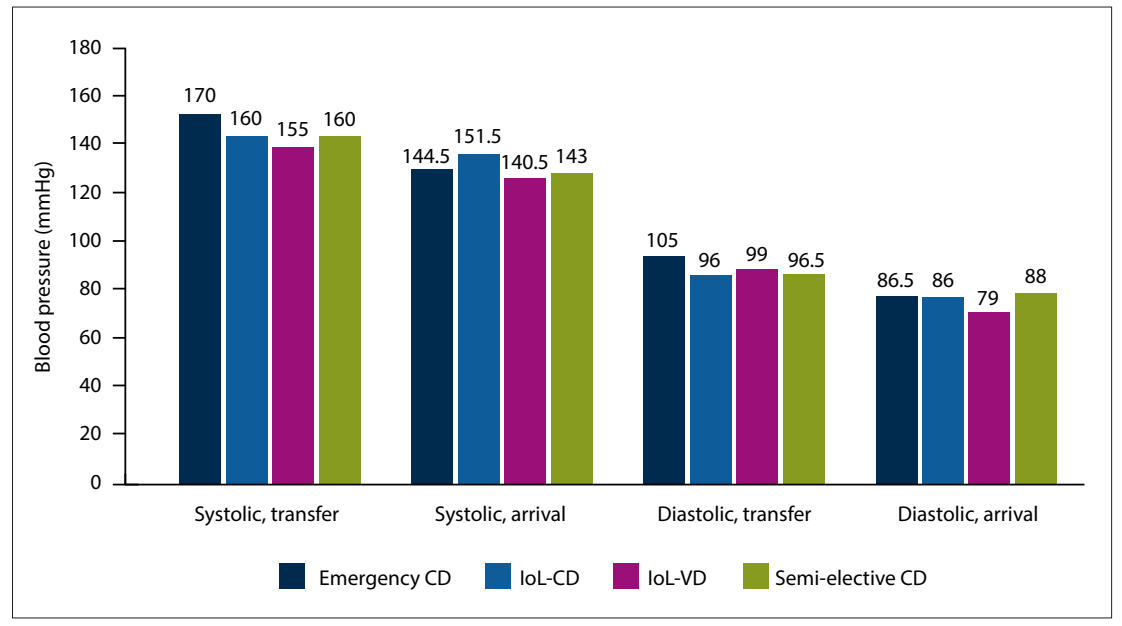

Fig. 1. Transfer and arrival blood pressure. $(C D=$ caesarean delivery; $I o L=$ induction of labour; $V D=$ vaginal delivery.)

Table 1. Demographic characteristics of the study population

\begin{tabular}{|c|c|c|c|c|c|c|}
\hline \multirow[b]{2}{*}{ Variables } & \multirow{2}{*}{$\begin{array}{l}\text { Maternal age (years), } \\
\text { median }\end{array}$} & \multirow{2}{*}{$\begin{array}{l}\text { BMI }\left(\mathrm{kg} / \mathrm{m}^{2}\right) \text {, } \\
\text { median }\end{array}$} & \multicolumn{2}{|c|}{ HIV status, $n(\%)$} & \multicolumn{2}{|c|}{ Parity, $n(\%)$} \\
\hline & & & Positive & Negative & Primiparous & Multiparous \\
\hline Emergency CD & 27.29 & 30.57 & $19(21.3)$ & $70(78.7)$ & $30(33.7)$ & $59(66.3)$ \\
\hline IoL-CD & 28.76 & 31.05 & $7(20.0)$ & $28(80.0)$ & $15(42.9)$ & $16(57.5)$ \\
\hline IoL-VD & 28.76 & 30.83 & $2(10.0)$ & $18(90.0)$ & $6(30.0)$ & $14(70.0)$ \\
\hline Semi-elective CD & 29.63 & 30.78 & $3(12.5)$ & $21(87.5)$ & $6(25.0)$ & $18(75.0)$ \\
\hline Median/total & 28.76 & 30.8 & $31(18.5)$ & $137(81.5)$ & $57(33.9)$ & $111(66.1)$ \\
\hline$p$-value & 0.7006 & 0.5136 & \multicolumn{2}{|c|}{0.6397} & \multicolumn{2}{|c|}{0.5488} \\
\hline
\end{tabular}




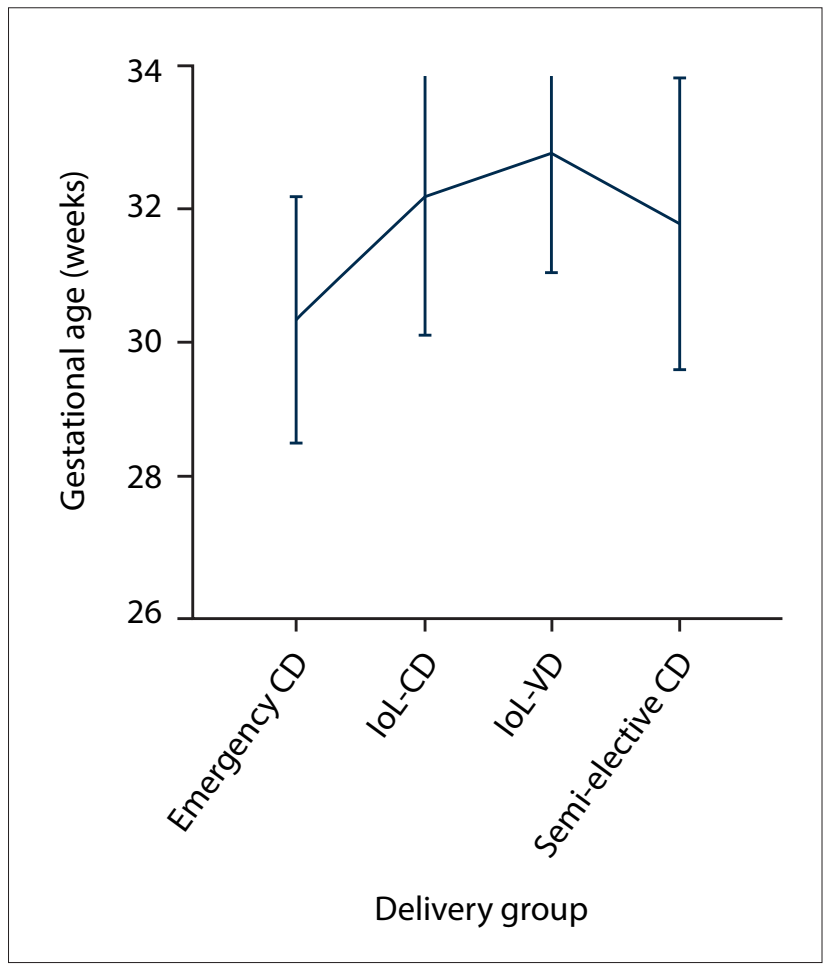

Fig. 2. Median gestational age at delivery decision. $(C D=$ caesarean delivery; $I o L=$ induction of labour; $V D=$ vaginal delivery.)

management of patients with EOPES), eclampsia and uncontrolled hypertension. Eleven patients presented with eclampsia and IoL was attempted in 8 , but only $1 \mathrm{VD}$ was achieved. Among the 39 women who were managed expectantly until 34 weeks 0 days, IoL was attempted in 29, with resultant VD in $13(45 \%)$.

\section{Bishop score}

Regarding the status of the cervix in the induction groups, 29 women had modified Bishop scores $>5$ and 13 had scores $>7$. Successful IoL resulting in VD was more likely with a more favourable Bishop score, although this was statistically insignificant $(p=0.2591)$ (Fig. 3 ).

\section{Previous CDs}

IoL was attempted in 5 patients with a previous CD; 2 had VDs and 3 required CDs, of which 2 were for failed IoL and 1 for fetal distress.

\section{Induction method}

Several different methods or agents were used for IoL. Prostaglandin E1 (PGE1), given orally, was used in 20 patients (36\%), resulting in 5 VDs and 15 CDs. This was the only agent used in 10 of the patients, and 9 of them required emergency CD. PGE1 used in combination with mechanical cervical dilatation (MCD) via insertion of an intracervical Foley catheter, followed by amniotomy, resulted in an additional 4 VDs and 5 CDs. Prostaglandin E2 (PGE2), as a vaginal preparation, was used in 21 patients (38\%), resulting in 7 VDs and 14 CDs. In all the VD cases where PGE2 was used initially, this was followed by MCD and amniotomy. Of the remaining 15 patients (27\%), 12 had MCD followed by amniotomy and oxytocin; VD was achieved in 6 of these patients. The last 3 patients who underwent IoL had favourable cervices, and direct amniotomies were performed in 2 of these cases, resulting in $1 \mathrm{CD}$ and $1 \mathrm{VD}$. One patient (HIV-positive) had oxytocin only, with a resultant $\mathrm{CD}$. Four of the 7 patients in whom IoL failed were HIVpositive, and early amniotomies were not performed.

\section{Fetal growth}

An EFW determined by ultrasound measurement was available in 155 of the 168 cases (Fig. 4). The EFW was plotted at $\leq 3$ rd centile in 37 of these cases (24\%), and between the 3rd and 10th centiles in 28 (18\%). Twenty-four $(65 \%)$ of the patients who plotted at $\leq 3$ rd centile required emergency CDs; 8 (22\%) were considered for IoL, of whom 6 required CD (Fig. 4). ${ }^{[8]}$

Fig. 5 reflects the birthweight $\mathrm{v}$. the accurate gestation and its plotting according to the centiles. ${ }^{[8]}$

\section{CD indications}

In the emergency CD group, all 89 patients had FHR tracings done with cardiotocography (CTG) that indicated fetal compromise requiring urgent delivery. Although all women in the IoL group $(n=55)$ had normal CTGs before IoL, 26 CTGs (47\%) became abnormal during the IoL and emergency CDs were required. Seven women had $\mathrm{CD}$ for failed inductions, and in 1 case the maternal condition did not allow IoL.

\section{UAD studies}

Thirty-seven (33\%) of the 113 UAD studies were abnormal for gestational age (Fig. 6). The UAD was considered abnormal if the resistance index was $\geq 95$ th centile for the gestational age, or $\geq 95$ th centile with periods of absent end-diastolic flow (AEDF), areas of AEDF, AEDF or reversal of end-diastolic flow. ${ }^{[9]}$

In the emergency $\mathrm{CD}$ group $(n=89), 24$ patients $(27 \%)$ had abnormal UADs; all required CD, indicated by abnormal CTGs and suspected fetal compromise. In the IoL group, UAD measurements were done for 41 of the 55 patients (75\%). There were $36(88 \%)$ with normal UADs and 5 with abnormal UADs. In the group with abnormal UADs, there was $1 \mathrm{VD}$, and $4 \mathrm{CDs}$ for suspected fetal compromise on the CTG. Of the 36 patients with normal UADs and CTGs in the induction group, 15 required CDs for fetal distress and 7 for failed IoL.

\section{Neonatal outcomes}

The median 1-minute Apgar score for the emergency CD group was $9 / 10$, as seen in 26 of the 88 available Apgar sets. For the IoL-CD group and the IoL-VD group, the median 1-minute Apgar score was also 9/10, and for the semi-elective CD group it was $8 / 10$. The emergency CD group had the most 1-minute Apgar scores $\leq 5$ ( $n=29$; $33 \%$ ), while there were 5 in the semi-elective CD group, 5 in the IoL$\mathrm{CD}$ group and none in the IoL-VD group. The emergency CD group had 95 -minute Apgar scores of $<5$, the semi-elective $\mathrm{CD}$ group had 3 , the IoL-CD group had 3 and the IoL-VD group had none. There were a total of 39 cases with initial Apgar scores of $\leq 5$ (44\%), and in 15 cases (17\%) the score remained at $\leq 5$ at the 5 -minute screening. This included 9 cases from the emergency CD group, 3 from the IoL$\mathrm{CD}$ group and 3 from the semi-elective group.

There were 2 stillbirths and 2 early neonatal deaths. In all these cases, emergency CD had been performed for fetal distress (fetal bradycardia or persistent fetal tachycardia). In the IoL-VD group there were no stillbirths or early neonatal deaths, and no cases with Apgar scores $\leq 5$.

\section{Hospital stay}

The hospital stay timeline (Fig. 7) shows that the differences in stay between all subgroups were significant. The median admissionto-delivery time between the subgroups was statistically different, with 11.38 days for the semi-elective patients, 15.55 days for the IoL-VD group, 8.34 days for the IoL-CD group and 4.93 days for the 


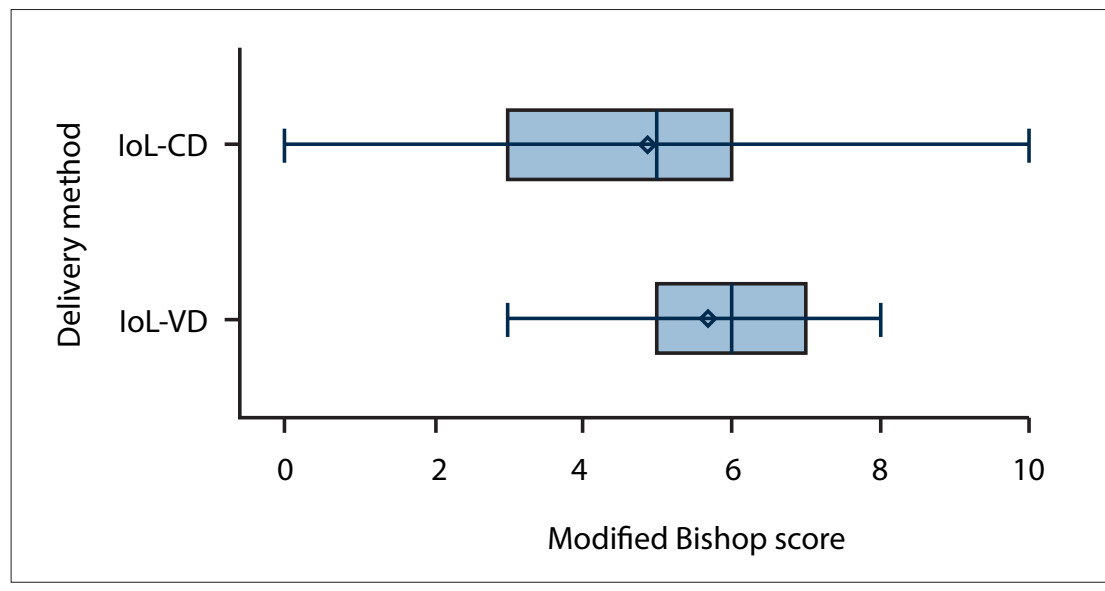

Fig. 3. Modified Bishop score and induction group delivery method. (IoL = induction of labour; $C D=$ caesarean delivery $; V D=$ vaginal delivery. $)$

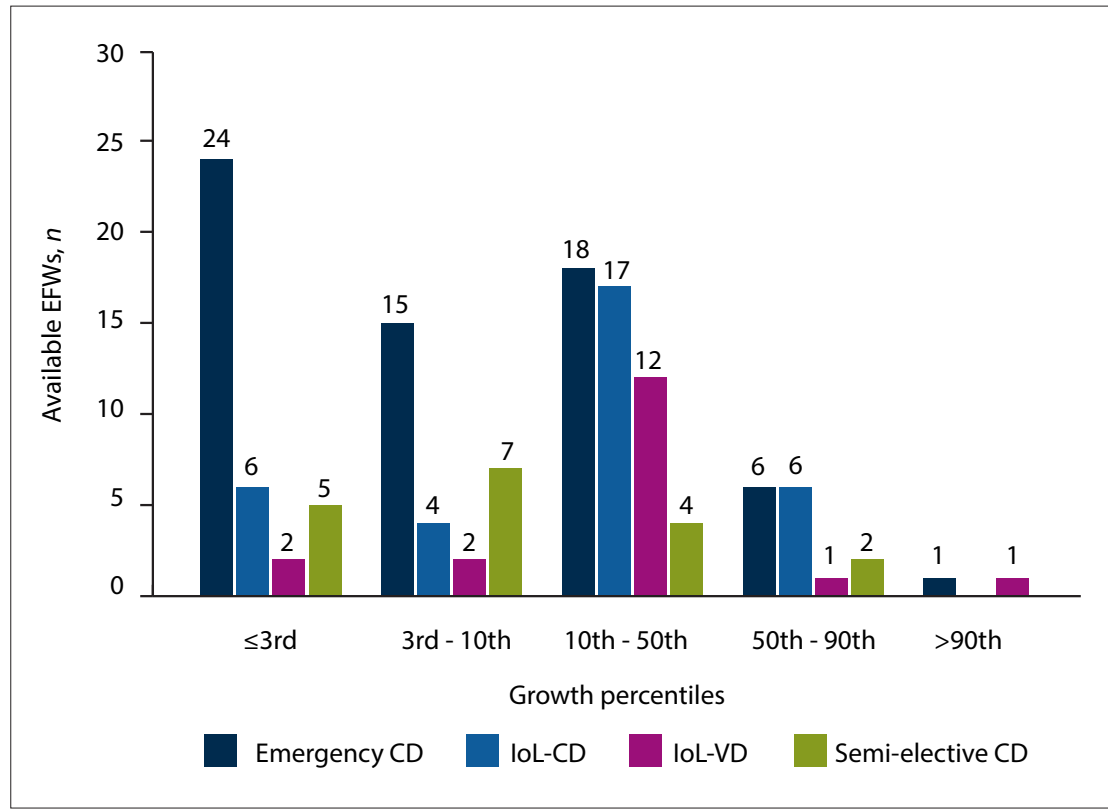

Fig. 4. EFW centile plotting. $(E F W=$ estimated fetal weight; $C D=$ caesarean delivery; $I o L=$ induction of labour; $V D=$ vaginal delivery.)

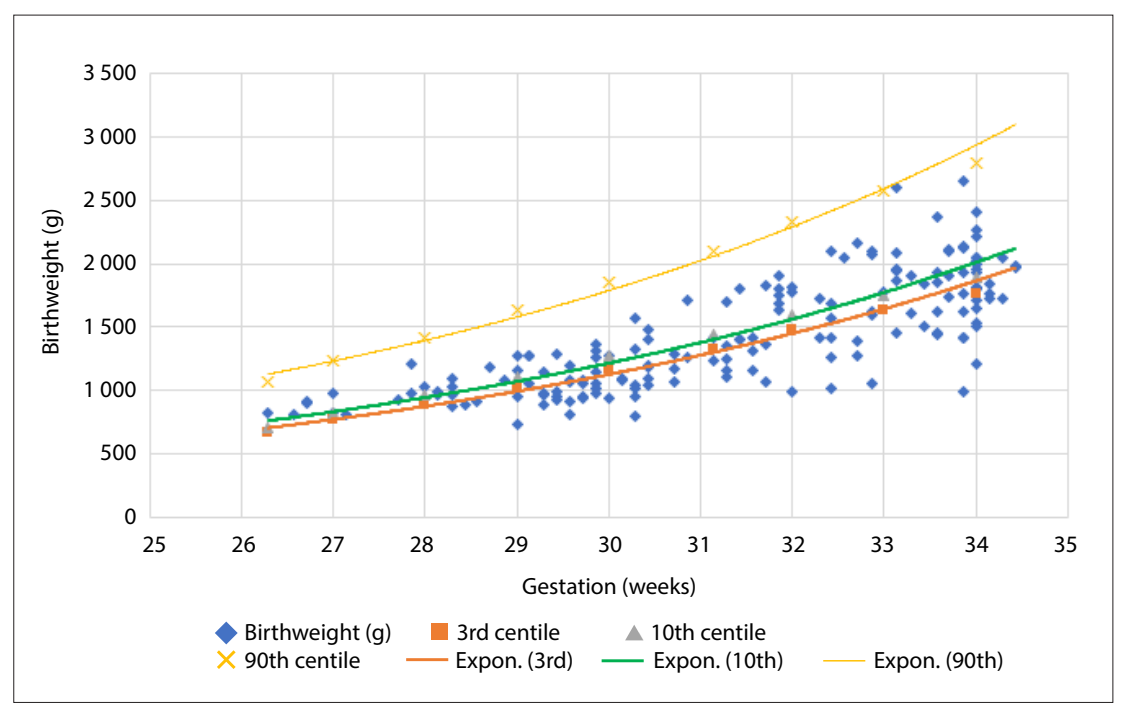

Fig. 5. Birthweight centile plotting. (Expon. (3rd), (10th) and (90th) = graphical representation of the distribution trends of birthweights less than the $3 r d, 10$ th and 90 th percentiles per gestational age.) emergency CD group $(p<0.001)$. The median decision-to-delivery time was longest for the IoL-CD group, and despite there not being a large difference in comparison with the 1.08 days for the semi-elective $\mathrm{CD}$ group and the 1.05 days for the IoL-VD group, the difference is significant if one considers the 0.16 days for an emergency $\mathrm{CD}(p<0.001)$.

\section{Expectant management}

Expectant management for inpatients is offered according to the institutional protocol for patients with EOPES where the maternal condition remains stable after admission. Of a group of 57 patients (34\%), 56 complied with this protocol and 1 declined. In the group who opted for inpatient expectant management, IoL was attempted in 23 patients (40\%), resulting in 12 VDs $(21 \%)(p=0.0383)$.

\section{Maternal complications}

Complications throughout hospital stay (antenatal and postnatal) are set out in Table 2.

The largest number of complications occurred in the emergency $\mathrm{CD}$ group, in association with the pre-eclampsia itself or due to a combination of pre-eclampsia and the surgical method of delivery. The complications in the IoL-VD group were due to the pre-eclamptic disease and not the delivery method. There were, however, 3 cases of puerperal sepsis and 1 wound haematoma in the IoL-CD group, compared with no cases of sepsis in the semi-elective $\mathrm{CD}$ and IoL-VD groups.

\section{Discussion}

In this EOPES study population, 20 patients (36\%) achieved successful VD following IoL. This success rate is significantly lower than previously found in an EOPES group (48\%) and with preterm inductions in general (57-80\%). ${ }^{[5,10]}$ Subgroup demographic differences (median maternal age, BMI, HIV status and parity) were minimal, promoting comparability.

A successful VD was more likely, although statistically insignificant, in multiparous women and with increased cervical favourability. These findings are supported by an SA study by Mashiloane and Moodley ${ }^{[11]}$ and concur with international evidence proving parity and cervical favourability to be the first- and second-strongest predictors of successful VD. ${ }^{[5]}$

The $40 \%$ IoL success rate in patients with a previous $\mathrm{CD}$ is in keeping with the overall success rate of $36 \%$ in this study, but lower than the $76.6 \%$ reported by Mardy et $a l^{[12]}$ in their analysis of vaginal birth 
after one previous lower-segment transverse caesarean section at 26 - 36 weeks' gestation. Their analysis was, however, not in a specific pre-eclamptic-only population.
Non-reassuring FHR patterns were found in $55 \%$ of their study population and inductions failed in $9.9 \%$ of their cases, ${ }^{[12]}$ similar to our findings.

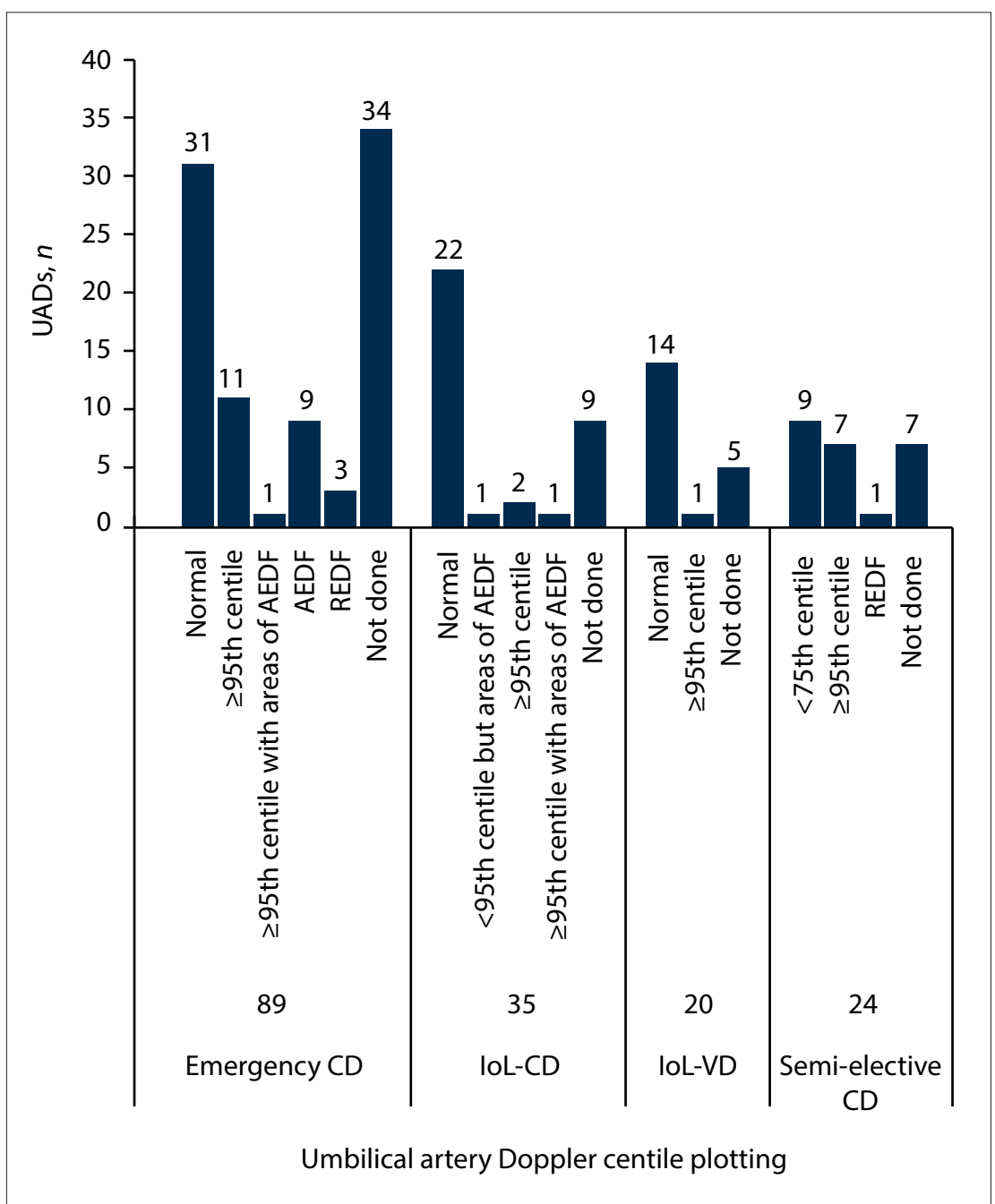

Fig. 6. UAD distribution. (UAD = umbilical artery Doppler; AEDF = absent end-diastolic flow; $R E D F=$ reversal of umbilical artery end-diastolic flow; $C D=$ caesarean delivery; IoL $=$ induction of labour; VD = vaginal delivery.)
The median gestational difference of 2 weeks 3 days $(p<0.001)$ between the emergency $\mathrm{CD}$ and IoL-VD subgroups represents the need for earlier urgent delivery in the more seriously affected cases, but also mirrors the findings by Feghali et al. ${ }^{[5]}$ of accelerated induction success with increased gestational age.

There were more attempted and successful IoLs among the patients who received expectant management for their preeclampsia. Patients who qualify for expectant management are more stable and have no immediate indications for delivery or maternal organ system dysfunction. Another institutional study that investigated the perinatal outcomes in an EOPES population who underwent expectant management reported attempted IoL in $30.7 \%$ of the women, with an $18.5 \%$ successful VD rate. ${ }^{[13]}$

The consecutive use of multiple induction agents was shown to be the superior approach (e.g. cervical priming by either prostaglandins, MCD or both, followed by amniotomy and oxytocin), and if fetal and maternal conditions allow it, the clinician should consider this step-by-step add-on process. Recent HIV viral load tests should be used to guide decisions regarding IoL with amniotomy. IoL with PGE1 should only be done with normal placental function, as its use was associated with the highest prevalence of CD.

The prevalence of pathological CTGs with suspected fetal distress is possibly a reflection of the disease burden characterised by abnormal placentation and poor fetal reserve. Evidence indicates that a normal UAD and CTG before IoL do not ensure successful IoL without fetal distress, complicating the predictability of fetal condition and compromise. The high probability of spontaneous fetal distress or

Table 2. Complications during hospital stay (antenatal and postnatal)

\begin{tabular}{|c|c|c|c|c|c|}
\hline Complications & Emergency CD, $n$ & IoL-CD, $n$ & IoL-VD, $n$ & Semi-elective CD, $n$ & Total, $N$ \\
\hline ICU/high-care admission & 5 & 5 & & 4 & 14 \\
\hline Eclampsia & 2 & 7 & 1 & 3 & 13 \\
\hline HELLP syndrome & 6 & 3 & 1 & 5 & 15 \\
\hline Pulmonary oedema & 3 & 3 & 1 & 3 & 10 \\
\hline Significant renal impairment (creatinine $\geq 125 \mathrm{mmol} / \mathrm{L}$ ) & 4 & 1 & & & 5 \\
\hline Resistant hypertension & 3 & & 1 & 1 & 5 \\
\hline Puerperal sepsis & 3 & 3 & & & 6 \\
\hline Wound haematoma & 3 & 1 & & 2 & 6 \\
\hline Massive transfusion ( $\geq 5$ units packed red cells) & 3 & & & & 3 \\
\hline Abruptio placentae & 8 & & & & 8 \\
\hline Visceral injury & 2 & & & & 2 \\
\hline Posterior reversible encephalopathy syndrome & & 1 & & & 1 \\
\hline Total & 42 & 24 & 4 & 18 & 88 \\
\hline
\end{tabular}




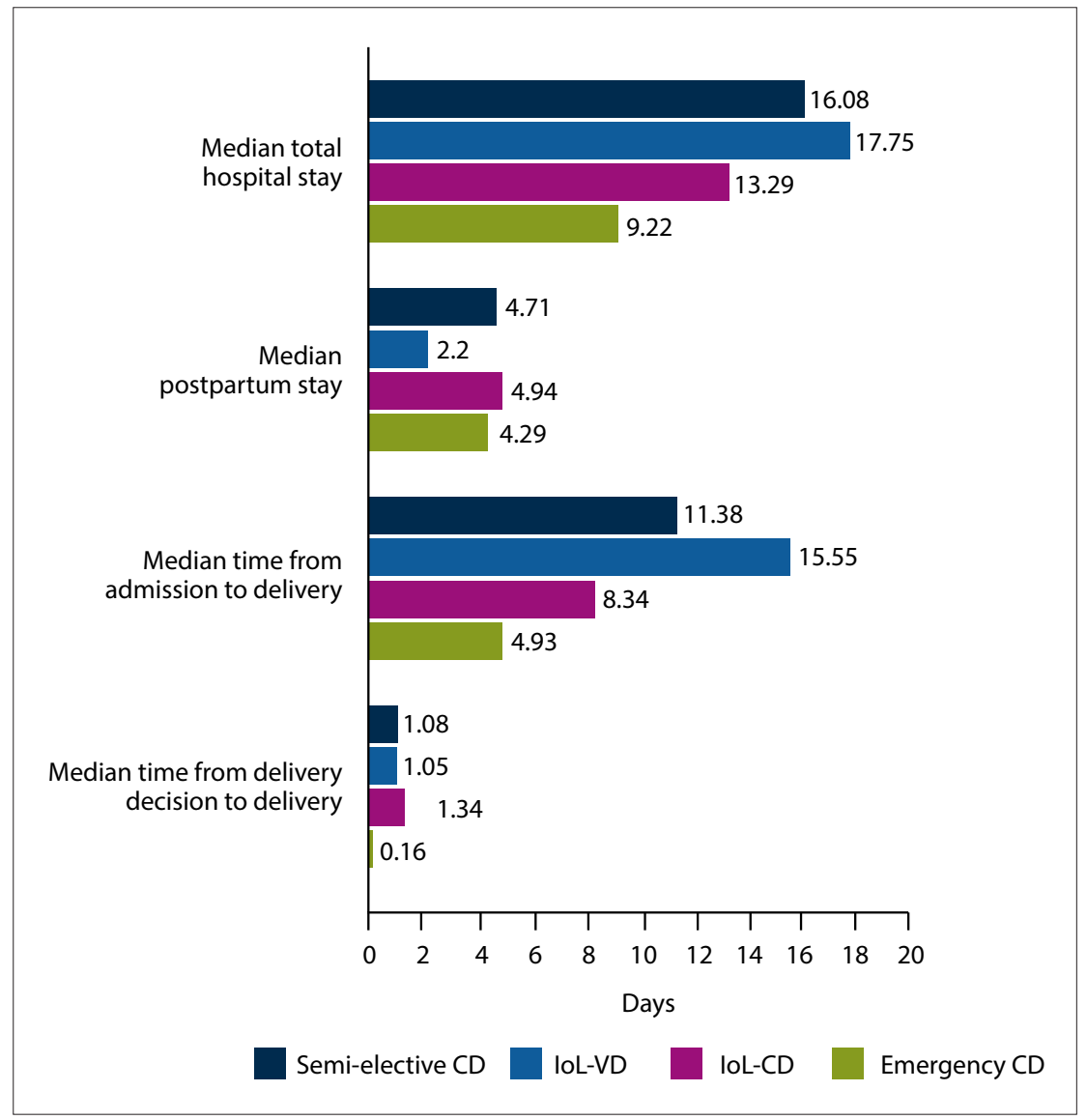

Fig. 7. Hospital stay timeline. $(C D=$ caesarean delivery; $I o L=$ induction of labour; $V D=$ vaginal delivery. $)$

fetal distress developing during labour also supports the use of $\mathrm{CD}$.

Hypertension and diabetes, even at term, increase the likelihood of $\mathrm{CD}$ due to associated failure to progress and non-reassuring FHR patterns. ${ }^{[3]}$ Patients with preterm EOPES are likely to have less favourable cervices, or poor placental function affecting the fetus, exacerbating this risk.

The median EFW was higher in the IoL groups than in the emergency and semielective $\mathrm{CD}$ groups. Clinician concern regarding impaired fetal growth or abnormal UADs could have influenced the allocation of cases to the semi-elective $\mathrm{CD}$ group. Clinician preference and experience contribute to the choice of delivery method. The estimated prevalence of $\mathrm{CD}$ is $70 \%$ in severe preeclampsia and potentially even higher in preterm pregnancy, as many obstetricians prefer $\mathrm{CD}$, even with reassuring fetal status. ${ }^{[14]}$

With an EFW $\leq 3$ rd centile, the prevalence of CD is high with or without IoL ( $75 \%$ and $78 \%$, respectively), and IoL is successful in 1 out of every 5 cases with an abnormal preinduction UAD. The UAD and EFW should therefore be carefully considered when deciding on the route of delivery. Figueras and Gratacos's ${ }^{[15]}$ fetal growth restriction stage-based management protocol deems
$\mathrm{CD}$ a reasonable option in cases with absent end-diastolic flow and recommends $\mathrm{CD}$ as the delivery method for reversal of umbilical artery end-diastolic flow.

The median Apgar score was good across all subgroups, with a score $>5$ at both 1 and 5 minutes in the IoL-VD group. In cases where IoL was appropriate and monitored carefully, there were no short-term adverse neonatal outcomes. Research conducted at another SA institution reported a significant increase in perinatal mortality between IoL-VD, elective $\mathrm{CD}$ and IoL-CD, but no significant difference in mortality between the two latter groups. ${ }^{[13]}$ This study also observed the postnatal course, and determined morbidity and mortality by including the development of respiratory distress syndrome, necrotising enterocolitis, pneumonia and sepsis (they included gestations $<28$ weeks). Future course of our study population, but this supersedes the purpose of our review.

The IoL-VD group had fewer complications than the other subgroups, possibly because this group had less severe disease. In 14 cases complications were secondary to $\mathrm{CD}$; no complications were attributed to the VD route alone, but rather to the disease process. research could be conducted on the neonatal

\section{Study strengths and weaknesses}

The subspecialist-led care with standardised protocols for management of pre-eclampsia and IoL in addition to the high prevalence of early-onset pre-eclampsia, contributes to the strengths of this study. Comparisons of conclusions were possible because data were obtained from both the induction and $\mathrm{CD}$ groups.

Variables with potential limiting effects on our research were different induction methods, clinician-chosen induction agents, the clinician's interpretation of the fetal condition, and the lack of a formal standardised definition for failed induction (thereby implying that the diagnosis remained the opinion of the clinician). Retrospective data collection could also be complicated by missing information for specific cases, and the smaller subgroup analysis numbers could negatively affect confidence intervals.

\section{Conclusions}

The large number of variables considered here emphasises the complexity of the decision regarding route of delivery in a preeclamptic patient. Successful IoL with VD as outcome was observed in 36\% of the EOPES population. VD was more likely with a fetus appropriately grown for gestation. In the presence of an abnormal UAD, significantly impaired growth (EFW $\leq 3$ rd centile) or eclampsia, the decision to induce should be considered carefully, as the likelihood of CD is very high.

Declaration. The research for this study was done in partial fulfilment of the requirements for SEO's MMed in Obstetrics and Gynaecology at Stellenbosch University.

Acknowledgements. We express our gratitude to our Stellenbosch University affiliates, the Department of Obstetrics and Gynaecology and Tygerberg Hospital for granting us the opportunity to conduct this observational study. Author contributions. All authors contributed to this article.

Funding. None.

Conflicts of interest. None.

1. Moodley J. Saving Mothers 2014 - 2016: Seventh Triennial Report on Confidential Enquiries into Maternal Deaths in South Africa: Short report. 2018. https://www.westerncape.gov.za/ assets/departments/health/saving_mothers_2014-16_-_short_ report.pdf (accessed 24 September 2019)

2. National Institute for Health and Care Excellence (NICE). Hypertension in pregnancy: Diagnosis and management. Clinical guideline [CG107]. 25 August 2010. https://www.nice. org.uk/guidance/cg107 (accessed 18 February 2018).

3. Vrouenraets FPIM, Roumen FJME, Dehing CJG, van den Akker ESA, Aarts MJB, Scheve EJT. Bishop score and risk of cesarean delivery after induction delivery after induction of labor in nulliparous women. Obstet

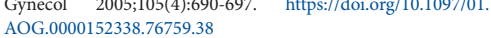


4. Heffiner LJ, Elkin E, Fretts RC. Impact of labor induction, gestational age, and maternal age on cesarean delivery rates. Obstet Gynecol 2003;102(2):287-293. https://doi.org/10.1016/S0029-7844(03)00531-3 delivery rates. Obstet Gynecol 2003;102(2):287-293. https:// doi.org/10.1016/S0029-7844(03)00531-3
5. Feghali M, Timofeev J, Huang C-C, et al. Preterm induction of labor: Predictors of vaginal delivery and . Feghali M, Timofeev J, Huang C-C, et al. Preterm induction of labor: Predictors of vaginal delivery and
labor curves. Am J Obstet Gynecol 2015;212(1):91.e1-91.e7. https://doi.org/10.1016/j.ajog.2014.07.035 6. Fawcus S, Pattinson RC, Moodley J, et al. Maternal deaths from bleeding associated with caesarean . Fawcus S, Pattinson RC, Moodley J, et al. Maternal deaths from bleeding associated with caesarean
delivery: A national emergency. S Afr Med J 2016;106(5):472-476. https://doi.org/10.7196/SAMJ.2016. delivery: A nation

7. Brown MA, Magee LA, Kenny LC, et al. Hypertensive disorders of pregnancy: ISSHP Classification, Diagnosis, and Management Recommendations for International Practice. Hypertension 1979;2018;72(1):24-43. https://doi.org/10.1161/HYPERTENSIONAHA.117.10803

8. Salomon LJ, Bernard JP, Ville Y. Estimation of fetal weight: Reference range at 20 - 36 weeks' gestation and comparison with actual birth-weight reference range. Ultrasound Obstet Gynecol 2007;29(5):550 555. https://doi.org/10.1002/uog.4019

9. Pattinson RC, Theron GB, Thompson ML, Lai Tung M. Doppler ultrasonography of the fetoplacental circulation - normal reference values. S Afr Med J 1989;76(11):623-625.

10. Nassar AH, Adra AM, Chakhtoura N, Gómez-Marín O, Beydoun S. Severe preeclampsia remote from term: Labor induction or elective cesarean delivery? Am J Obstet Gynecol 1998;179(5):1210-1213. https://doi.org/10.1016/S0002-9378(98)70133-4
11. Mashiloane $\mathrm{CD}$, Moodley J. Induction or caesarean section for preterm pre-eclampsia? J Obstet Gynaecol 2002;22(4):353-356. https://doi.org/10.1080/01443610220141245

12. Mardy AH, Ananth CV, Grobman WA, Gyamfi-Bannerman C. A prediction model of vaginal birth after cesarean in the preterm period. Am J Obstet Gynecol 2016;215(4):513.el-313.e7. https://doi. org/10.1016/.jajog.2016.05.039

13. Hall DR, Odendaal HJ, Kirsten GF, Smith J, Grove D. Expectant management of early onset, severe pre-eclampsia: Perinatal outcome. Br J Obstet Gynaecol 2000;107(10):1258-1264. https://do org/10.1111/j.1471-0528.2000.tb11617.x

14. Amorim MMR, Souza ASR, Katz L. Planned caesarean section versus planned vaginal birth for severe pre-eclampsia. Cochrane Database Syst Rev 2017, Issue 10. Art. No.: CD009430. https://doi. org/10.1002/14651858.CD009430.pub2

15. Figueras F, Gratacos E. An integrated approach to fetal growth restriction. Best Pract Res Clin Obstet Gynaecol 2017;38:48-58. https://doi.org/10.1016/j.bpobgyn.2016.10.006

Accepted 4 December 2020. 